\title{
A Study on the Relationship between Accrued Income and Earnings Quality of Listed Companies in the Hotel Industry
}

\author{
Liu Huiyue ${ }^{1, a}$, Hu Chenyan ${ }^{2, b, *}$ \\ 1Shenzhen Tourism College of Jinan University, Shenzhen, Guangdong, China \\ 2Shenzhen Tourism College of Jinan University, Shenzhen, Guangdong, China \\ aliu_hy@sz.jnu.edu.cn,bmelody.hcy@foxmail.com \\ ${ }^{*}$ Corresponding author
}

Keywords: Hotel industry listed companies, Accounts receivable, Earnings quality, Relevance.

\begin{abstract}
As a creditor's right of enterprises, accrued income directly influences the actual earnings level of enterprises. Based on the seasonal data from 2009Q1 - 2015Q3, by selecting the relevant indicators of accounts receivable as the indicators of measuring accrued income, an empirical test on the relevance of accounts receivable and earnings quality of listed companies in China's hotel industry is carried out. According to the study, we can draw conclusions below: the size of accounts receivable is positively related to earnings quality; the turnover rate of accounts receivable is positively related to earnings quality; the difference between the growth rate of operating income and the growth rate of accounts receivable is positively related to the earnings quality; hotel class listed companies' accounts receivable accounts for a higher proportion of assets, and the growth rate of accounts receivable is generally higher than the growth rate of operating income, but the average turnover rate of accounts receivable is within a reasonable range. Therefore, enterprises may be appropriate to relax the credit limit, establish the collection system strictly, strengthen the recovery and management of accounts receivable.
\end{abstract}

\section{酒店业上市公司应计收入与盈余质量关系研究 \\ 刘慧悦 ${ }^{1, a}$, 胡晨燕 $2, b,{ }^{*}$ \\ 1暨南大学深圳旅游学院, 深圳, 广东, 中国 \\ 2暨南大学深圳旅游学院, 深圳, 广东, 中国 \\ aliu_hy@sz.jnu.edu.cn, bmelody.hcy@foxmail.com \\ *通讯作者}

关键词: 酒店行业上市公司; 应收账款; 盈余质量; 关联性

中文摘要. 应计收入作为企业债权, 直接影响企业实际的盈余水平。本文基于2009年第一季 度至2015年第三季度的季报数据, 选用应收账款的相关指标作为衡量应计收入的指标, 对我 国酒店行业上市公司的应收账款和盈余质量的关联性进行实证检验。研究结果证明: 应收账 款规模与盈余质量正相关; 应收账款周转率与盈余质量正相关; 营业收入增长率与应收账款 增长率的差额与盈余质量正相关; 酒店类上市公司的应收账款占资产比重较高, 应收账款的 增长速度普遍高于营业收入的增长速度, 但应收账款平均周转速度在合理范围内。因此, 可 适当放宽挂账的额度, 严格建立催收制度, 加强应收账款的回收与管理。

\section{一、引言}

随着社会主义市场经济的进一步推进，商业信用在市场中的运用日益广泛，在资金贵乏 
的状况下，㻌销有利于企业销售产品和开拓市场。因此，企业应计收入规模日益增大，作为 企业重要的流动资产, 应收账款对企业的盈余质量会产生重要的影响。企业如何在日常经营 活动中有效地管理应收账款, 成为目前企业财务管理的重要问题。同时, 投资者比以往更加 关注上市公司的盈余质量问题。由于企业当期应收账款规模的增大，会直接导致当期账目利 润的减少, 削弱了主营业务利润以及每股收益对企业经营状况的评价作用。在一定程度上, 应收账款的存在导致了所有者权益真实性的降低。

近年来, 盈余质量的研究是国内外学者研究的热点。盈余质量被称为 “利润质量” 或者 “收益质量”。Baginski, Lorek, Willimger 和 Branson(1999) ${ }^{[1]}$ 认为盈余持久性的影响因素包 括了公司规模、产品类型、准入壁垒和资本集中度，通过建立回归模型并比较回归模型的调 整后的判定系数, 来研究由这四个因素评价的盈余质量的持久性, 研究结果表明, 高阶 ARlMA 模型明显比低阶模型具有更好的拟合优度。Cahan, Emanuel 和 Sun $(2009)^{[2]}$ 发现投资者保护程 度高的国家盈余质量和盈余价值的相关性更强，并且盈余质量和盈余价值与国家的信息透明 度正相关。Peterson, Schmardebeck 和 Wilks $(2015)^{[3]}$ 认为会计的一致性与盈余质量有关, 对于 盈余质量的衡量选取了盈余的持续性、可预测性、收益质量、可操控性的应计项四个指标。 而国内关于盈余质量的研究, 始于上世纪 90 年代, 大部分的研究集中在 2005 年前后, 主要 集中在其内涵研究和评价体系构建这两个方面上。郭永清 $(2000)^{[4]}$ 研究认为, 公司的盈余质量 是一项重要标准，根据过去的盈余质量可以评价公司当期的盈余质量。盈余质量变化通常被 解释为公司经营环境、财务环境和公司未来前景已经发生变化。王秀丽和张新民 $(2005)^{[5]}$ 提出 高质量的利润结构应具有如下特点: 利润自身结构的协调性、利润结构与企业发展战略的符 合性、利润结构与主营业务的核心性、利润结构与资产结构的匹配性、利润结构与对应的现 金流量结构的趋同性。吴德军和唐国平 $(2009)^{[6]}$ 的研究对盈余质量的评价方法进行归纳, 将常 用的盈余质量方法归纳为市场评价法、应计项目评价法、线下项目评价法、综合度量方法等 四类方法。

其中, 对于应计收入与盈余质量关联性的研究也备受学者的关注。Beneish(1999) ${ }^{[7]}$ 的研究 说明应计收入的增长是企业推进盈余管理的驱动因素。Chan 和 Jegadeesh(2004) ${ }^{[8]}$ 通过研究证 实可操纵应计项会导致当期盈余上升，未来盈余下降。随着人们对盈余质量的关注度日渐提 高, 我国学者也开始了对应收账款与盈余质量相关性进行研究, 并取得一定成果。张惠忠 $(2006)^{[9]}$ 通过探讨我国上市公司盈余质量, 发现当应收账款增幅大于主营业务收入增幅时, 坏 账发生的比率增大。韩晓明 $(2011)^{[10]}$ 的研究证明, 应计项目变化趋势与会计制度变化密切相关, 表明应计项目的价值相关性与持续性一致, 能够较好地反映会计信息质量的高低。林东杰和 刘梦宁 $(2013)^{[11]}$ 提出应收款 (包括应收账款和其他应收款) 坏账准备计提异常与盈余质量显著 负相关, 其中, 应收账款坏账准备计提异常与盈余质量负相关, 但不显著; 其他应收款坏账 准备计提异常与盈余质量显著负相关，更容易被企业用来操纵盈余。

酒店类上市公司应计收入主要以应收账款为主。①在以信用卡消费为主流的当下，酒店业 上市公司不可避免应收账款占流动资产比重较大的现状, 若应收账款不能按时收回, 将会对 企业的资金周转产生严重影响, 从而影响企业经营与运作的有效性。本文研究侧重于对我国 酒店行业上市公司应收账款与盈余质量的相关性的分析。虽然应收账款对于盈余质量的影响 越来越被学者所关注。但是, 目前为止, 对于应计收入与盈余质量相关性的研究较少, 主要 还是针对我国上市公司整体而进行的研究，但不同行业经营方式不同，应计收入的种类与特 点不同, 对盈余质量带来的影响也有区别。因此通过研究酒店行业上市公司应收账款与盈余 质量的相关性，可以丰富应收账款视角下的盈余质量评价体系的研究。 


\section{二、研究假设与模型设定}

\section{1.酒店行业应收账款的特点}

本文的研究以应收账款的相关指标衡量酒店行业上市公司应计收入水平。应收账款，作 为企业的一项债权, 是企业在正常的经营过程中, 由销售商品、产品、提供劳务等业务引起 的，应向购买单位收取的款项，包括应由购买单位或接受劳务单位负担的税金、代购买方垫 付的各种运杂费等 (许雅胥, 2011) ${ }^{[12]}$ 。酒店行业发生应收账款的原因主要有两种：第一，商 业竞争。在社会主义市场经济剧烈的竞争下，企业普遍采用㻌销的手段来扩大销售。为应对 竞争而实行㻌销所引起的应收账款, 是一种商业信用。例如, 星级酒店会给予签有挂账协议 的企业一定金额的挂账的权利。第二，销售和收款的时间差距。对于一些企业来说由于货款 结算需要时间, 且时间长短受结算手段影响, 因此其商品交易与收到货款的时间往往不一致, 从而导致了企业需要先垫付的应收账款。例如酒店客人通过信用卡结账, 银行统一结算时间 往往在下一个工作日，这就导致了酒店业的信用卡应收账款。

酒店行业上市公司应收账款可分为宾客账 (Guest Ledger)、外客账 (City Ledger) 和信用卡 (Credit Card)。其主要特点为：应收账款规模较大; 长住客宾客账的应收账款账龄一般较长; 由客人拒付而引起的应收账款回收难度较大。应收账款规模、应收账款周转率、应收账款增 长速度和应收账款账龄均会对盈余质量造成不同程度上的影响，从而导致企业利润表账面利 润虚高、所有者权益真实性的降低，同时削弱了每股收益对公司业绩进行评价的作用，成为 公司操控利润的重要手段之一。

\section{2.评价酒店行业盈余质量的指标选取}

企业盈余质量具有真实性、现金保障性、安全性、持续性和成长性五个特点。酒店类上 市公司的盈余质量受到多方面因素的影响，包括行业竞争环境、酒店行业的周期性波动、经 营杜杆和财务杜杆、企业的利润来源以及企业的信用政策。盈余质量是指是会计信息质量的 核心内容, 它反映了盈余信息满足不同利益相关者制定决策时信息需求的能力 (袁克利, 2011 $)^{[13]}$ 。因此, 本文根据盈余质量的内涵与特点, 结合数据可获取性, 选取如下五个指标, 合成评价酒店行业盈余水平的综合指标 $E Q I$ :

表 1 酒店行业盈余质量 $E Q I$ 合成的指标说明

\begin{tabular}{|c|c|c|}
\hline 基础指标 & 指标计算 & 指标说明 \\
\hline 利润率 $\left(\mathrm{Y}_{1}\right)$ & 营业利润/利润总额 & 反映盈余质量的持续性 \\
\hline $\begin{array}{c}\text { 利润经营现金流量比 } \\
\left(\mathrm{Y}_{2}\right)\end{array}$ & 经营活动现金净流量/营业利润 & 体现盈余质量的现金保障性 \\
\hline 利润增长率 $\left(\mathrm{Y}_{3}\right)$ & $\begin{array}{c}\text { (本季度营业收入-上年同季度营业收入)/ } \\
\text { 上年同季度营业收入 }\end{array}$ & 反映盈余质量的成长性 \\
\hline 经营现金流量比率 $\left(\mathrm{Y}_{4}\right)$ & 经营活动现金净流量/期末流动负债 & 反映盈余质量的安全性 \\
\hline 税后利润率 $\left(\mathrm{Y}_{5}\right)$ & 扣除非经常性损益后的净利润/净利润 & 衡量盈余质量的真实性 \\
\hline
\end{tabular}

\section{3.研究假设}

本文将从应收账款的角度，对酒店类上市公司的盈余质量进行实证分析，考察两者之间 的相关关系。将应收账款拆分为三个指标, 分别为应收账款规模、应收账款周转率及营业收 入增长率与应收账款增长率差额。因此我们提出以下假设:

假设 (1) 酒店行业应收账款规模、应收账款周转率, 主营业务增长率与应收账款增长率 的差额与 $E Q I$ 综合评价指数之间存在线性关系。

应收账款规模，指应收账款占总资产的比率。应收账款规模越大，表明引起账面利润与 实际现金流入的差额越大, 则公司的支付能力、偿债能力、经营能力, 发展后劲不足, 盈余 质量也越低。因此, 本文提出假设:

假设 Q : 酒店行业应收账款规模与盈余质量负相关。 
应收账款周转率是营业收入与应收账款平均余额的比率。它反映了企业在一个会计期间 内回收应收款项的能力。应收账款周转率越高, 表明企业收回应收款项的时间越短, 资金利 用率越高, 盈余质量越好。因此, 本文提出以下假设:

假设 (3): 酒店行业应收账款周转率与盈余质量正相关。

应收账款增长速度，一般越低越好。若企业应收账款增长率远高于营业收入增长率，则 表示企业很可能采取了过于宽松的信用政策, 这将会导致企业应收账款规模增大, 甚至是账 龄变长, 应收账款回收难度增大, 坏账风险提高, 从而导致了企业利润表账面利润虚高, 实 际利润降低。因此，本文做出以下假设:

假设 (4): 酒店行业营业收入增长率与应收账款增长率的差额与盈余质量正相关。文中提 到的变量及其定义如下:

表 2 主要变量及其意义

\begin{tabular}{c|c|c|c}
\hline & 变量名称 & 变量含义 & 变量解释 \\
\hline $\begin{array}{c}\text { 被解释变 } \\
\text { 量 }\end{array}$ & $E Q I$ & 盈余质量综合评价得分 & 运用变异权数综合评价法计算得到 \\
\hline \multirow{3}{*}{ 解释变量 } & $X_{1}$ & 应收账款规模 & 应收账款占总资产的比率 \\
\cline { 2 - 4 } & $X_{2}$ & 应收账款周转率 & 营业收入与应收账款平均余额的比率 \\
\cline { 2 - 4 } & $X_{3}$ & 营业收入增长率与应收账款增长率差额 & 应收账款增长速度 \\
\hline
\end{tabular}

\section{4.模型设定}

根据前文分析，本文设定面板数据的多元线性回归模型如下:

$$
E Q I_{i t}=C_{i t}+\beta_{i t} X_{i t}+\mu_{i t}(i=1,2, \ldots \ldots, N) \quad(t=1,2, \ldots \ldots, T)
$$

其中 $N$ 表示个体截面成员的个数, $T$ 表示每个截面成员的观测时期总数, 参数 $C_{i t}$ 表示模 型的常数项, $\beta_{i t}$ 表示对应于解析变量向量 $X_{i t}$ 的 $k \times 1$ 维系数向量, $k$ 表示解析变量的个数, $\mu_{i t}$ 表示随机误差项。

\section{三、实证研究结果}

\section{1.数据来源}

2016 年 1 月，同花顺财经（http://www.0033.com/）证券板块中，以客房和餐饮收入为主 营业务收入的酒店餐饮类上市公司共有 10 家, 剔除 ST 公司两家, 以及在样本截取时间内曾 是 ST 公司的企业一家, 最终共有样本数 7 家, 它们分别是: 大东海 A, 全聚德, 华天酒店, 锦江股份，岭南控股，金陵饭店，西安饮食。其时间区间均为 2009 年第一季度至 2015 年第 三季度，共 27 期的季报数据。

\section{2.变异权数法计算的酒店行业盈余质量综合指标}

为了衡量样本企业的盈余指标综合指标, 本文选取了变异权数法对样本的盈余评价得分 进行计算。利用已经获得的 5 个指标计算指标的均值和标准差及标准离差率, 其中标准离差 率反映了各指标的相对变异程度, 然后对其进行归一化处理得到各指标权数, 由此获得盈余 质量综合评价指数。

\section{3.描述性统计}

对表 2 中的变量进行描述性统计, 统计结果如下表 3 : 
表 3 主要变量描述性统计

\begin{tabular}{c|c|c|c|c}
\hline 变量 & 极小值 & 极大值 & 均值 & 标准差 \\
\hline$E Q I$ & -8.480 & 8.466 & 0.982 & 1.335 \\
\hline$X_{1}$ & 0.004 & 0.064 & 0.020 & 0.011 \\
\hline$X_{2}$ & 2.101 & 98.338 & 18.242 & 17.287 \\
\hline$X_{3}$ & -4.856 & 3.942 & -0.141 & 0.781 \\
\hline
\end{tabular}

从被解释变量来看, $E Q I$ 极大值为 8.466 , 极小值为 -8.480 , 极大值与极小值之间存在 比较大的差距，反映了不同公司之间，或者同公司不同季度的盈余质量水平是有较大的差异。

从解释变量来看, 应收账款规模均值约为 $20 \%$, 说明在酒店类上市公司中, 应收账款占 资产的比重较大。应收账款周转率极小值为 2.101 , 极大值为 98.338 , 反映了酒店类行业不 同公司之间的应收账款的平均周转速度差异较大，并且在时间上也有较大的波动性。营业收 入增长率与应收账款增长率差额均值为负值, 说明酒店类上市公司应收账款的增长率普遍高 于营业收入的增长率。

\section{4.模型的检验及调整}

由于面板数据的回归分析要求解析变量之间相互独立, 所以在进行回归分析之前需要先 对 3 个解析变量进行相关性分析。经分析, 可以看出, $X_{1}, X_{2}, X_{3}$ 之间并无呈现高的相关性, 因此三个自变量可以同时与解析变量进行多元线性回归分析。

由于面板数据模型常常存在非平稳性特征, 因此需要对样本数据进行单位根检验, 确定 数据是否平稳。面板数据单位根检验有很多种方法, 本文为平衡面板数据, 故只采用了一种 面板数据单位根检验方法, 即 Fisher-ADF 检验。经检验, 可以看出, 四个变量都是平稳的, 因此，可以进一步进行协整检验。

本文选择了 Pedroni 检验和 Kao 检验两种方法来对解析变量进行协整检验。根据检验结 果，可以近似地认为，变量间存在协整关系，即变量间具有长期的均衡关系。

因此, 本文采用的面板数据模型如下, 接下来会进一步对该模型进行混合、固定效应和 随机效应的选择。同时, 由于本研究截面数量小于时间段数, 因此, 在做面板数据的混合、 固定效应和随机效应的选择时, 在时间效应 (Period) 模型和截面效应 (Cross-Section) 之间选取 时间效应 (Period) 模型。

$$
E Q I_{i t}=C_{i t}+\beta_{i t} X_{i t}+\mu_{i t}(i=1,2, \ldots \ldots, N) \quad(t=1,2, \ldots \ldots, T)
$$

其中 $N$ 表示个体截面成员的个数, $T$ 表示每个截面成员的观测时期总数, 参数 $C_{i t}$ 表示模 型的常数项, $\beta_{i t}$ 表示对应于解析变量向量 $X_{i t}$ 的 $k \times 1$ 维系数向量, $k$ 表示解析变量的个数, $\mu_{i t}$ 表示随机误差项。

为选择合适的模型，首先，通过 $\mathrm{F}$ 统计量检验判断使用混合模型还是固定效应模型; 第 二步, 通过 Hausman 检验判断使用固定效应模型还是随机效应模型。经过 $\mathrm{F}$ 统计量检验, 结 果说明 $\mathrm{F}$ 统计量检验拒绝原假设 $\mathrm{H} 0$, 因此使用固定效应模型优于混合模型。经过 Hausman 检验，结果说明 Hausman 检验拒绝原假设 H0，因此本文采用固定效应模型。

\section{5.实证检验与结果分析}

表 4 给出了面板数据回归方程系数表: 
表 4 面板数据回归方程系数表

\begin{tabular}{|c|c|c|c|c|}
\hline 变量 & 相关系数 & 标准误 & $\mathrm{t}-$ 统计量 & $P$ 值 \\
\hline 应收账款规模 $\left(X_{1}\right)$ & 9.714 & 5.298 & 1.833 & 0.069 \\
\hline 应收账款周转率 $\left(X_{2}\right)$ & 0.012 & 0.004 & 3.457 & 0.001 \\
\hline $\begin{array}{c}\text { 营业收入增长率与应收账 } \\
\text { 款增长率差额 }\left(X_{3}\right)\end{array}$ & 0.284 & 0.082 & 3.470 & 0.001 \\
\hline$C$ & 0.590 & 0.135 & 4. 366 & 0.000 \\
\hline
\end{tabular}

回归方程如下:

$$
E Q I=9.714 X_{1}+0.012 X_{2}+0.284 X_{3}+0.590+\mu_{i t}(i=1,2, \ldots \ldots ., N) \quad(t=1,2, \ldots \ldots ., T)
$$

其中 $\mu_{i t}$ 为回归方程随机误差值。

通过面板数据回归方程系数表，可得出酒店行业上市公司应收账款与盈余质量相关性的 分析结果:

(1) $X_{1}$ 的相关系数为 9.714 , 证明应收账款规模与盈余质量正相关, 假设 $\mathrm{H} 2$ 不成立。由 相关系数可见，应收账款规模的增大还会带来盈余质量的大幅度提高; 由标准误看出, 应收 账款规模均数抽样误差较大; 由 $\mathrm{t}$-统计量看出, 其样本平均值在靶值附近, 说明样本平均值 与靶值无显著差异; 由 $P$ 值看出，应收账款规模对盈余质量没有显著影响。

(2) $X_{2}$ 的相关系数为 0.012 , 应收账款周转率与盈余质量正相关, 假设 $\mathrm{H} 3$ 成立。由相关 系数可见, 应收账款周转率的提高会给盈余质量带来正向的影响, 但影响幅度并不大。由标 准误看出, 应收账款规模均数抽样几乎没有误差; 由 $\mathrm{t}$-统计量看出, 其样本平均值稍偏离靶 值, 说明样本平均值与靶值有轻微差异; 由 $P$ 值看出, 应收账款规模对盈余质量有显著影响。

(3) $X_{3}$ 的相关系数为 0.284 , 营业收入增长率与应收账款增长率差额与盈余质量正相关, 假设 $\mathrm{H} 4$ 成立。由相关系数可见, 营业收入增长率与应收账款增长率差额的增长能带来约 0.2 倍的盈余质量的提高。由标准误看出, 应收账款规模均数抽样几乎没有误差; 由 $\mathrm{t}$-统计量看 出, 其样本平均值偏离靶值, 说明样本平均值与靶值稍有差异; 由 $P$ 值看出, 应收账款规模 对盈余质量有显著影响。

(4)上述验证分析过程证明, $X_{1}, X_{2}, X_{3}$ 均可以与盈余质量综合评价得分 $(E Q I)$ 构建线性 回归的关系，假设 H1 成立。

因此，实证研究的结果充分说明了在我国酒店行业上市公司中，盈余质量会受到应收账 款规模、应收账款周转率和营业收入增长率与应收账款增长率差额的影响。

\section{四、结论与建议}

本文从应收账款的角度，对酒店类上市公司的盈余质量进行实证分析，得出以下结论：

1. 对于酒店行业上市公司，应收账款规模与盈余质量正相关。一方面，酒店为扩大销售， 增加酒店竞争力, 大规模的运用赊销手段所产生的应收账款促进了酒店销售的提升与利润额 的增长; 另一方面, 随着电子支付手段的推进, 越来越多的客人使用信用卡结账从而造成了 应收账款，但这种由于入账发生时间与银行方结算时间不一致而造成的应收账款一般能在下 一个工作日银行结算后冲抵，在长期不会造成对盈余质量的负面影响。

2. 酒店的应收账款周转率越高, 其盈余质量越高。应收账款周转率高, 反映了酒店宾客 拖账欠账的行为少, 外客账的回款效率高, 以及酒店收银员和内审员比较负责而很少出现信 用卡少刷漏刷的情况, 这些都有效的保障了酒店运作的现金流动性, 对盈余质量的提高具有 正面的影响。

3. 营业收入增长率与应收账款增长率的差额越大，盈余质量越高。一定量的㻌账制度有 利于促进酒店客房和会议的销售, 带来营业收入的增长, 但应加强应收账款的回收与管理, 来控制应收账款的过快增长, 以保持营业收入增长率与应收账款增长率的较大差额, 来保障 
酒店方的盈余质量。

4. 酒店类上市公司的应收账款占资产比重较高, 应收账款的增长速度普遍高于营业收入 的增长速度, 这些问题都会加大酒店方收回账款的难度, 增加酒店的营运成本, 应该给予重 视。但目前研究的样本酒店的应收账款平均周转速度都在合理范围内, 尚不会导致营运所需 现金流不足等问题。

应收账款作为企业重要的流动资产，对企业的盈余质量会产生重要的影响。因此应从应 收账款的角度提高酒店类上市公司的盈余质量, 首先, 在制定严格的挂账及应收账款催收款 制度的前提下，可适当放宽挂账的额度，促进酒店的销售，来促进盈余质量的提升; 其次, 建立相关制度提高酒店应收账款周转率，保障足够的流动资金来支持企业的正常运营; 最后, 应加强应收账款的回收与管理，来控制应收账款的过快增长，以保持营业收入增长率与应收 账款增长率的较大差额, 来保障酒店方的盈余质量。另外, 应当建立严格的应收账款催收制 度。一方面有利于酒店类上市公司提高资产管理能力, 另一方面也有利于为投资者提供更有 效的决策信息。

\section{注释:}

酒店类上市公司应收账款是指在日常经营活动中，为了扩大酒店销售，增加酒店竞争力，而 允许宾客㻌账消费所产生的宾客挂账、外客挂账等应收取而未收取款项，以及客人通过信用 卡结算，信用卡消费的银行到账时间与酒店入账时间不一致所形成的应收账款等。

基金项目: 本研究得到国家社会科学基金青年项目(14CJY004)及中央高校基本科研业务费专 项资金 (22614817)的资助。感谢匿名审稿专家的宝贵建议，当然文责自负。

作者简介: 刘慧悦(1981 年一), 女, 汉, 吉林长春人, 暨南大学深圳旅游学院, 讲师, 经济 学博士, 研究方向为旅游财务分析。

胡晨燕(1996 年一), 女, 侗, 四川内江人, 暨南大学深圳旅游学院。通信人联系 邮箱: melody.hcy@foxmail.com, 联系电话: 15889490236 ; 样刊邮寄地址: 广东省深圳市南 山区侨香路 6 号暨南大学深圳旅游学院; 邮编：518053。

\section{References}

[1] Baginski S.P.,K.S.Lorek,G.L.Willinger and B.C.Branson. The relationship between economic characteristic and alternative annual earnings persistence measure [J]. Accounting Review, 1991, (1): $105-120$.

[2] Cahan S F, Emanuel D, Sun J, et al. The effect of earnings quality and country-level institutions on the value relevance of earnings[J]. Review of Quantitative Finance and Accounting, 2009, 33(4): 371-391.

[3] Peterson K, Schmardebeck R, Wilks T J, et al. The Earnings Quality and Information Processing Effects of Accounting Consistency[J]. The Accounting Review, 2015.

[4] Guo Yongqing. The Analysis of Earnings Quality[J]. Finance and Economics, 2000, (1): 69-74.

[5] Wang Xiuli, Zhang Xinmin. The Analysis on the Characteristics and Quality of Enterprise Profit Structure[J]. Accounting Research, 2005, (9): 63-68.

[6] Wu Dejun, Tang Guoping. The Measurement Method of Earnings Quality in Empirical Study[J]. Journal of Zhongnan University of Economics and Law, 2009, (4): 93-98.

[7] MD Beneish. Incentives and Penalties Related to Earnings Overstatements That Violate GAAP[J] . Accounting Review A Quarterly Journal of the American Accounting Association, 1999, 74(4): 425-457. 
[8] Chan K, Jegadeesh N, Sougiannis T, et al. The Accrual Effect on Future Earnings[J]. Review of Quantitative Finance and Accounting, 2004, 22(2): 97-121.

[9] Zhang Huizhong. Briefly Discussion on the Analysis of Earnings Quality of Listed Companies[J]. Journal of Lixin Accounting College, 2006, (3), 13-16.

[10]Han Xiaoming. Accruals, Cash Flow and Accounting Earnings[J]. Financial Research, 2011(9): 77-80.

[11]Lin Dongjie, Liu Mengning. The Relationship between Bad Debts Provision and Corporate Earnings Quality- - Based on the Empirical Evidence of the Chinese Listed Companies[J]. Journal of Nanjing Audit University, 2013, (5): 67-74.

[12]Xu Yaxi. Corporate Receivables Management and Risk Prevention[J]. Communication of Finance and Accounting, 2011, (35), 72-73.

[13] Yuan Keli. Research on Earnings Quality (II) - - Determinants and Economic Consequences of Earnings Quality[J]. Financial Economy, 2011, (24), 63-66. 